\title{
Different Mixing Scenerio of Quasi-Degenerate Neutrino with Charged Lepton Correction
}

\author{
Mrinal Kumar Das \\ Department of Physics, Tezpur University, Tezpur, India \\ Email: mkdas@tezu.ernet.in
}

Received August 16, 2012; revised September 15, 2012; accepted September 22, 2012

\begin{abstract}
Theoretically, in order to achieve non-zero $\theta_{13}$ a little deviation from Tribimaximal Mixing (TBM) pattern is needed, especially on $\theta_{13}$ without perturbing the atmospheric and solar mixing angles. In this work we computed the neutrino mixing angles by disturbing the $\theta_{13}$ as well as $\theta_{12}$ in Bimaximal (BM) and Hexagonal mixing (HM) using non-diagonal charged lepton mass. Considering the standard form of mass texture which satisfies TBM we have shown the quasidegenerate nature of neutrino. This quasi degenerate type of mass matrix for BM and HM is then used to calculate the deviated mixing pattern which are consistent with recent neutrino oscillation data.
\end{abstract}

Keywords: Neutrino Mixing, Neutrino Masses, Charged Lepton Correction

\section{Introduction}

Recent solar, atmospheric, reactor, and accelerator neutrino experiments have provided concrete evidence that neutrinos are massive and they change their flavors during propagation. In the standard neutrino oscillation picture three active neutrinos are involved, with masssquared differences of order $10^{-5}$ and $10^{-3} \mathrm{eV}^{2}$. The deficit in the neutrino flux from solar and atmospheric neutrinos have confirmed that at least two neutrinos should have non-zero masses. The mixing pattern and the tiny neutrino masses makes the explanation of the origin of neutrino masses and leptonic flavor mixing one of the most prominent problems in the particle physics. The mixing of lepton flavors is described by a $3 \times 3$ unitary matrix, whose nine elements are commonly parametrized in terms of three rotation angles and three CP-violating phases. Defining three unitary rotation matrices in the complex planes one can express neutrino mixing in terms of three rotations:

$$
\begin{aligned}
& U_{23}=\left(\begin{array}{ccc}
1 & 0 & 0 \\
0 & c_{23} & s_{23} \mathrm{e}^{-\mathrm{i} \delta_{23}} \\
0 & -s_{23} \mathrm{e}^{\mathrm{i} \delta_{23}} & c_{23}
\end{array}\right), \\
& U_{13}=\left(\begin{array}{ccc}
c_{13} & 0 & s_{13} \mathrm{e}^{-\mathrm{i} \delta_{13}} \\
0 & 1 & 0 \\
-s_{13} \mathrm{e}^{\mathrm{i} \delta_{13}} & 0 & c_{13}
\end{array}\right),
\end{aligned}
$$

$$
U_{12}=\left(\begin{array}{ccc}
c_{12} & s_{12} \mathrm{e}^{-\mathrm{i} \delta_{12}} & 0 \\
-S_{12} \mathrm{e}^{\mathrm{i} \delta_{12}} & c_{12} & 0 \\
0 & 0 & 1
\end{array}\right),
$$

where $c_{i j}=\cos \theta_{i j}$ and $s_{i j}=\sin \theta_{i j} \quad$ with $1 \leq i, j \leq 3$. Using these three rotations, expression of neutrino mixing with standard parameterizations become

$$
U=U_{23} U_{13} U_{12} P
$$

where $U$ is known as Pontecorvo-Maki-NakagawaSakata (PMNS) matrix and can be written as

$$
\begin{aligned}
& U=U_{\text {PMNS }} \\
& =\left(\begin{array}{ccc}
c_{13} c_{12} & c_{13} s_{12} & s_{13} e^{-i \delta} \\
-c_{23} s_{12}-c_{12} s_{13} s_{23} \mathrm{e}^{\mathrm{i} \delta} & c_{12} c_{23}-s_{12} s_{13} s_{23} \mathrm{e}^{\mathrm{i} \delta} & c_{13} s_{23} \\
s_{12} s_{23}-c_{12} s_{13} c_{23} \mathrm{e}^{\mathrm{i} \delta} & -c_{12} s_{23}-c_{23} s_{13} s_{12} & c_{13} c_{23}
\end{array}\right) P,
\end{aligned}
$$

where $P=\operatorname{Diag}\left(\mathrm{e}^{\mathrm{i} / \beta_{1}}, \mathrm{e}^{\mathrm{i} / 2}, 1\right)$ is a diagonal phase matrix which contains two non-trivial Majorana phases of CP violation. This also involves just three irremoveable physical phases $\delta_{i j}$. In this parameterizations the Dirac phase $\delta$ which enters the CP odd part of neutrino oscillation probabilities is given by $\delta=\delta_{13}-\delta_{23}-\delta_{12}$. The recent global fit [1] to the various neutrino experimental data has given the following mixing angle values and non-zero of $\theta_{13}$. 


$$
\begin{aligned}
& \sin ^{2} \theta_{23}=0.466_{-0.058}^{+0.073} \\
& \sin ^{2} \theta_{12}=0.312_{-0.018}^{+0.019}
\end{aligned}
$$

and

$$
\sin ^{2} \theta_{13}=0.016 \pm 0.010 .
$$

In view of above mixing angles it is clear that the Tribimaximal mixing (TBM) [2-4],

$$
U_{\mathrm{PMNS}}=\left(\begin{array}{ccc}
\sqrt{\frac{2}{3}} & \frac{1}{\sqrt{3}} & 0 \\
-\frac{1}{\sqrt{6}} & \frac{1}{\sqrt{3}} & \frac{1}{\sqrt{2}} \\
-\frac{1}{\sqrt{6}} & \frac{1}{\sqrt{3}} & -\frac{1}{\sqrt{2}}
\end{array}\right),
$$

can give the close description to the neutrino oscillation as well with a minor correction in $\theta_{13}$. The predictions of Equation (9) viz. $\sin ^{2} \theta_{23}=\frac{1}{2}$ and $\sin \theta_{13}=0$ $\sin ^{2} \theta_{12}=\frac{1}{3}$, are consistent with atmospheric and solar neutrino oscillation with minor correction in $\theta_{13}$. As the global analysis of neutrino data has provided hints for non-zero $\theta_{13}$ [5-7]. The first observational hint for nonzero $\theta_{13}$ has come from the $\mathrm{T} 2 \mathrm{~K}$ experiment [8]. After T2K experiment MINOS experiment also disfavor the $\theta_{13}=0$ [9]. To achieve non-zero $\theta_{13}$ theoretically is an interesting topic in neutrino physics. Now, recent analysis on neutrino mixing it is proposed by many papers [10-12] that apart from TBM there are some other mixing pattern like Bimaximal mixing (BM), Hexagonal mixing (HM) and Tetragonal mixing can also give the alternative description of neutrino mixing with correction. Among these, tribimaximal mixing gives very close description of the experimentally found mixing angles when the best fit values are presumed.

In the proposed work we give a description of neutrino mixing which is achieved in the framework of bimaximal, tribimaximal and hexagonal mixing with the help of charged lepton correction. In the present paper, we take non-diagonal charged lepton mass in expression of $m_{L L}$ given by Equation (11). This correction can be realised in the Pontecorvo-Maki-Nakagawa-Sakata (PMNS) matrix in the form of $U_{\mathrm{PMNS}}=U=U_{l}^{\dagger} U_{v}$, where $U_{l}$ diagonalise the charged lepton mass matrix $m_{L R}$. The matrix $U_{l}$ is considered as Cabibbo like mixing matrix as already been discussed in a recent work [13] for TBM case. This correction will deviate the solar mixing $\theta_{12}$ and CHOOZ mixing $\theta_{13}$ to the experimental range in case $\mathrm{BM}$ and $\mathrm{HM}$ without disturbing the $\theta_{23}$.

The seesaw mechanism is used to construct neutrino mass models e.g.: Quasi-degenerate, Normal Hierarchi- cal $(\mathrm{NH})$ and Inverted Hierarchical (IH), are discussed in our earlier work $[14,15]$. Out of these three models which model can give good prediction to the neutrino oscillation is also a topical question in recent neutrino physics. In this work we analyze on Quasi-degenerate neutrino mass model ( $\mathrm{NH}$ and $\mathrm{IH}$ ) with different mixing pattern as discussed above. In Section 2, we shown neutrinos are quasi-degenerate $(\mathrm{NH}$ and $\mathrm{IH})$ in nature considering neutrinos are mixed tribimaximally. Next in Section 3, different neutrino mass models with BM and HM considering neutrinos are quasi-degenerate in nature are discussed. Then with the help of charged lepton correction how these mixing can predict new mixing which are consistent with recent experimental data along with non-zero $\theta_{13}$ is also discussed. And in the final section i.e. in Section 4 , there are some concluding remarks.

\section{Quasi-Degenerate Neutrino}

As mentioned in the introduction neutrino mass eigenvalues can have three kinds of pattern normal, inverted hierarchical and quasi-degenerate. We know, from neutrino oscillations that the neutrino mass pattern is nondegenerate. The pattern is hierarchical, if $m_{1} \ll \Delta m_{\odot}^{2}$, $m_{1}$ is smallest neutrino mass and $\Delta m_{\odot}^{2}$ is solar mass square difference. When $m_{1} \ll \Delta m_{\text {atm }}^{2}$, the pattern is inverted hierarchical, where $m_{3}$ is the smallest mass. In the quasi-degenerate case both the ordering may be possible.

The most popular neutrino mixing which gives the TBM form is given by Equation (9). This can be generated with two generators $S$ and $T$ of $A_{4}$ symmetry, one of which gives charged lepton mass matrix diagonal and other gives the invariant neutrino mass matrix $m_{\mathrm{TBM}}^{v}$ [16], where $m_{\mathrm{TBM}}^{v}$ is given by

$$
m_{\mathrm{TBM}}^{v}=\left(\begin{array}{ccc}
A & B & B \\
B & \frac{1}{2}(A+B+D) & \frac{1}{2}(A+B-D) \\
B & \frac{1}{2}(A+B-D) & \frac{1}{2}(A+B+D)
\end{array}\right),
$$

gives $m_{1}=A+B, \quad m_{2}=A-2 B$ and $m_{3}=D$. The mass matrix given by Equation (10) is constructed on the basis of the type I see-saw mechanism,

$$
m_{L L}=m_{L R} M_{R R}^{-1} m_{L R}^{T},
$$

where $m_{L R}$ is diagonal. The present neutrino oscillation data gives the following information for the mass square difference.

$$
\begin{aligned}
& 7.05 \times 10^{-5} \mathrm{eV}^{2} \leq \Delta m_{21}^{2} \leq 8.34 \times 10^{-5} \mathrm{eV}^{2}, \\
& 2.07 \times 10^{-3} \mathrm{eV}^{2} \leq \Delta m_{31}^{2} \leq 2.75 \times 10^{-3} \mathrm{eV}^{2},
\end{aligned}
$$


with the following best fit values

$$
\begin{aligned}
& \Delta m_{21}^{2}=7.65 \times 10^{-5} \mathrm{eV}^{2}, \\
& \Delta m_{31}^{2}=2.40 \times 10^{-3} \mathrm{eV}^{2} .
\end{aligned}
$$

Using $m_{1}, m_{2}$ and $m_{3}$ from Equation (10), mass square differences and the sum of the three mass eigenvalues $\left(m_{\text {cosmo }}=\sum_{i} v_{i}<0.61 \mathrm{eV}\right)$ can be expressed in terms of $A, B$ and $D$. By solving these three equations in terms of $A, B$ and $D$ values of $m_{1}, m_{2}$ and $m_{3}$ are calculated with MATHEMATICA and listed in the Table 1 , which are quasi-degenerate in nature.

These values of $A, B$ and $D$ are now used to construct the neutrino mass matrices with the help of Equation (10) for $\mathrm{NH}$ and IN case are:

- Normal hierarchical:

$$
m_{L L}=\left(\begin{array}{ccc}
-0.052487 & 0.105217 & 0.105217 \\
0.105217 & 0.10905 & -0.05632 \\
0.105217 & -0.05632 & 0.10905
\end{array}\right)
$$

\section{- Inverted hierarchical:}

$$
m_{L L}=\left(\begin{array}{ccc}
0.1063024 & -0.0001196 & -0.0001196 \\
-0.0001197 & 0.100396 & 0.005786 \\
-0.00011966 & 0.005786 & 0.100396
\end{array}\right)
$$

After Diagonalising the above mass matrices, calculated mass square differences and mixing angles are listed in the Table 2, which shows TBM property.

\section{Deviation from Original Mixing Pattern with Charged Lepton Correction}

From analysis of recent neutrino oscillation parameter it is observed that neutrino mixing is very close to TBM pattern. Deviation from TBM is recently reported [13, 17], where important corrections are incorporated to make the mixing angle match with the experimental data. Different possible alternatives to this TBM are, e.g. Bimaximal, Trimaximal, Hexagonal mixing as well golden ration angles, discussed in recent work [10]. This alterna- tive mixing pattern can also arrive in the experimental range with corrections. In this section we try to produce a mixing pattern, from Bimaximal, Hexagonal as well as Tribimaximal mixing with charged lepton correction, which is consistent with recent neutrino oscillation data. In general, the Pontecorvo-Maki-Nakagawa-Sakata (PMNS) matrix with charged lepton correction can be expressed as product of two unitary matrices as

$$
U=U_{l}^{\dagger} U_{v}
$$

where $U_{v}$ diagonalizes the neutrino mass matrix as

$$
m_{L L}(\text { diagonal })=U_{v} m_{L L} U_{v}^{\mathrm{T}},
$$

$U_{l}$ diagonalizes the charged lepton mass matrix as

$$
m_{L R}(\text { diagonal })=U_{l} m_{L R} U_{l}^{\mathrm{T}} .
$$

In our earlier work $[14,15]$, in construction of $m_{L L}$, using seasaw I we were considered the dirac mass $M_{L R}$ is diagonal, which can be considered as either charged lepton or up quark type. However, a general form of the Dirac neutrino mass matrix is given by

$$
m_{L R}=\left(\begin{array}{ccc}
\lambda^{m} & 0 & 0 \\
0 & \lambda^{n} & 0 \\
0 & 0 & 1
\end{array}\right)
$$

where $m_{f}$ corresponds to $m_{\tau} \tan \beta$ for $(m, n)=(6,2)$ in the case of charged lepton and $m_{t}$ for $(m, n)=(8,4)$ in the case of up-quarks. Here $\lambda$ can pick value between 0.104 and 0.247 for the Dirac neutrino mass matrix. In this pattern the $U_{\text {PMNS }}$ matrix given by Equation (14) doesnot involve $U_{l}$. In the present analysis we use non-diagonal $m_{L R}$ in seesaw I and due to this reason one has to use the contribution $U_{l}$ in the PMNS matrix. We construct a deviated neutrino mixing matrix $U$ i.e. PMNS matrix using Equation (14), where charged leptons mass matrices are considered to be non-diagonal. This predicts mixing angles, are consistent with recent oscillation data. To construct $U$ we consider $U_{v}$ in three different forms e.g. BM, HM as well as TBM and the

Table 1. Values of $A, B, D, m_{1}, m_{2}, m_{3}$.

\begin{tabular}{ccccccc}
\hline Type & $A$ & $B$ & $D$ & $m_{1}$ & $m_{2}$ & $m_{3}$ \\
\hline $\mathrm{NH}$ & -0.052487 & 0.105217 & 0.16537 & 0.157704 & 0.157947 & 0.16537 \\
$\mathrm{IH}$ & 0.106302 & -0.0001197 & 0.09461 & 0.106182 & 0.106541 & 0.09461 \\
\hline
\end{tabular}

Table 2. Mass square differences and mixing angles.

\begin{tabular}{cccccc}
\hline Type & $\Delta m_{21}^{2}\left[10^{-5} \mathrm{eV}^{2}\right]$ & $\Delta m_{31}^{2}\left[10^{-3} \mathrm{eV}^{2}\right]$ & $\sin ^{2} \theta_{12}$ & $\sin ^{2} \theta_{23}$ & $\sin \theta_{13}$ \\
\hline $\mathrm{NH}$ & 7.67 & 2.39 & 0.37 & 0.5 & 0 \\
$\mathrm{IH}$ & 7.68 & 2.40 & 0.33 & 0.5 & 0 \\
\hline
\end{tabular}


elements of matrix $U_{l}$ are calculated considering it is a Cabibbo-Kobayashi-Maskawa like mixing i.e. [13]:

$$
\sin \theta_{12}^{l}=\lambda, \quad \sin \theta_{23}^{l}=a \lambda^{2}, \quad \sin \theta_{13}^{l}=b \lambda^{3},
$$

where $\lambda$ varies between 0.104 to 0.247 and $a, b$ varies between 0.2 to 5 . From Equation (18) calculated $\theta^{l}$ are used to construct the elements of matrix $U_{l}$.

\subsection{Hexagonal Mixing}

The standard form of hexagonal mixing matrix is

$$
U_{v}^{\mathrm{HM}}=\left(\begin{array}{ccc}
\frac{\sqrt{3}}{\sqrt{2}} & \frac{1}{2} & 0 \\
-\frac{1}{2 \sqrt{2}} & \frac{\sqrt{3}}{2 \sqrt{2}} & -\frac{1}{\sqrt{2}} \\
-\frac{1}{2 \sqrt{2}} & \frac{\sqrt{3}}{2 \sqrt{2}} & \frac{1}{\sqrt{2}}
\end{array}\right)
$$

which can predict $\sin ^{2} \theta_{12}=\frac{1}{4}, \sin ^{2} \theta_{23}=\frac{1}{2}$ and $\sin ^{2} \theta_{13}=0$. Using Equation (19) one can also construct $m_{L L}$ for $\mathrm{HM}$ as

$$
\begin{aligned}
m_{L L}^{\mathrm{HM}}= & \left(\begin{array}{ccc}
\frac{\sqrt{3}}{\sqrt{2}} & \frac{1}{2} & 0 \\
-\frac{1}{2 \sqrt{2}} & \frac{\sqrt{3}}{2 \sqrt{2}} & -\frac{1}{\sqrt{2}} \\
-\frac{1}{2 \sqrt{2}} & \frac{\sqrt{3}}{2 \sqrt{2}} & \frac{1}{\sqrt{2}}
\end{array}\right)^{T}\left(\begin{array}{ccc}
m_{1} & 0 & 0 \\
0 & m_{2} & 0 \\
0 & 0 & m_{3}
\end{array}\right) \\
& \cdot\left(\begin{array}{ccc}
\frac{\sqrt{3}}{\sqrt{2}} & \frac{1}{2} & 0 \\
-\frac{1}{2 \sqrt{2}} & \frac{\sqrt{3}}{2 \sqrt{2}} & -\frac{1}{\sqrt{2}} \\
-\frac{1}{2 \sqrt{2}} & \frac{\sqrt{3}}{2 \sqrt{2}} & \frac{1}{\sqrt{2}}
\end{array}\right),
\end{aligned}
$$

and has a texture

$$
m_{L L}^{\mathrm{HM}}=\left(\begin{array}{ccc}
A & B & B \\
B & \left(A+\sqrt{\frac{8}{3}} B+D\right) & \left(A+\sqrt{\frac{8}{3}} B-D\right) \\
B & \left(A+\sqrt{\frac{8}{3}} B-D\right) & \left(A+\sqrt{\frac{8}{3}} B+D\right)
\end{array}\right),
$$

where mass eigenvalues are

$$
m_{1}=A-\frac{2}{3} B, \quad m_{2}=A+\sqrt{6} B, \quad m_{3}=D .
$$

\subsection{Bimaximal Mixing}

The standard form of bimaximal mixing is written as:

$$
U_{v}^{\mathrm{BM}}=\left(\begin{array}{ccc}
\frac{1}{\sqrt{2}} & \frac{1}{\sqrt{2}} & 0 \\
-\frac{1}{2} & \frac{1}{2} & -\frac{1}{\sqrt{2}} \\
-\frac{1}{2} & \frac{1}{2} & \frac{1}{\sqrt{2}}
\end{array}\right)
$$

which predicts $\sin ^{2} \theta_{12}=\frac{1}{2}, \sin ^{2} \theta_{23}=\frac{1}{2}$ and $\sin ^{2} \theta_{13}=0$. Using Equation (23) for the quasi-degenerate case one can construct neutrino mass model for BM case as:

$$
\begin{aligned}
m_{L L}^{\mathrm{HM}}= & \left(\begin{array}{ccc}
\frac{1}{\sqrt{2}} & \frac{1}{\sqrt{2}} & 0 \\
-\frac{1}{2} & \frac{1}{2} & -\frac{1}{\sqrt{2}} \\
-\frac{1}{2} & \frac{1}{2} & \frac{1}{\sqrt{2}}
\end{array}\right)^{T}\left(\begin{array}{ccc}
m_{1} & 0 & 0 \\
0 & m_{2} & 0 \\
0 & 0 & m_{3}
\end{array}\right) \\
& \cdot\left(\begin{array}{ccc}
\frac{1}{\sqrt{2}} & \frac{1}{\sqrt{2}} & 0 \\
-\frac{1}{2} & \frac{1}{2} & -\frac{1}{\sqrt{2}} \\
-\frac{1}{2} & \frac{1}{2} & \frac{1}{\sqrt{2}}
\end{array}\right),
\end{aligned}
$$

the texture has form [15]

$$
m_{L L}^{\mathrm{BM}}=\left(\begin{array}{ccc}
\left(1-2 \delta_{1}-2 \delta_{2}\right) & -\delta_{1} & -\delta_{1} \\
-\delta_{1} & -\delta_{2} & 1-\delta_{2} \\
-\delta_{1} & 1-\delta_{2} & -\delta_{2}
\end{array}\right) m_{o}
$$

where mass eigen values are

$$
\begin{aligned}
& m_{1}=\left(1-\delta_{1}-\sqrt{3} \delta_{1}-2 \delta_{2}\right) m_{o}, \\
& m_{2}=\left(1-\delta_{1}+\sqrt{3} \delta_{1}-2 \delta_{2}\right) m_{o}, \\
& m_{3}=m_{o}
\end{aligned}
$$

The $m_{1,2,3}$ values can be calculated for HM as well as $\mathrm{BM}$ case, which are quasi-degenerate in nature using similar procedure adopted in Section 2 for TBM case. For HM $A=0.104669, B=-0.128304, D=0.210183$ and for $\mathrm{BM} \delta_{1}=7.2 \times 10^{-5}, \delta_{2}=3.9 \times 10^{-3}$ are calculated using MATHEMATICA. Values of mass square differences and mixing angles for BM \& HM are given in Table 3. The expression for $m_{o}$ is defined as [15] $m_{o}=m_{f}^{2} / v_{o}$, where $v_{o}=6.6 \times 10^{12}$.

Now elements of $U_{v}$ are taken from Equation (19) and (23) for HM and BM respectively. Then using Equation (18) choosing the suitable value for $\lambda, a$ and $b$ elements of $U_{l}$ have been calculated. Finally deviated matrix $U$ is constructed using the following equation: 
Table 3. Values of $\theta_{12}, \theta_{23}, \theta_{13}$ and mass scales.

\begin{tabular}{cccccc}
\hline Type & $\Delta m_{21}^{2}\left[10^{-5} \mathrm{eV}^{2}\right]$ & $\Delta m_{31}^{2} / \Delta m_{23}^{2}\left[10^{-3} \mathrm{eV}^{2}\right]$ & $\sin ^{2} \theta_{12}$ & $\sin ^{2} \theta_{23}$ & $\sin \theta_{13}$ \\
\hline Bimaximal Mixing & 7.91 & 2.50 & 0.50 & 0.50 & 0 \\
Hexagonal Mixing & 7.67 & 2.40 & 0.25 & 0.50 & 0 \\
\hline
\end{tabular}

Table 4. Values of $\theta_{12}, \theta_{23}$ and $\theta_{13}$.

\begin{tabular}{ccccccc}
\hline Mixing type & $a$ & $b$ & $\lambda$ & $\sin ^{2} \theta_{12}$ & $\sin ^{2} \theta_{23}$ & $\sin \theta_{13}$ \\
\hline Bimaximal Mixing & 0.25 & 0.25 & 0.18 & 0.26 & 0.44 & 0.047 \\
Hexagonal Mixing & 0.3 & 0.3 & 0.16 & 0.31 & 0.42 & 0.061 \\
Tri-Bimaximal Mixing & 0.3 & 0.3 & 0.2 & 0.29 & 0.49 & 0.064 \\
\hline
\end{tabular}

$$
U=U_{\mathrm{PMNS}}=\left(\begin{array}{ccc}
c_{13}^{l} c_{12}^{l} & c_{13}^{l} s_{12}^{l} & s_{13}^{l} \\
-c_{23}^{l} s_{12}^{l}-c_{12}^{l} s_{13}^{l} s_{23}^{l} & c_{12}^{l} c_{23}^{l}-s_{12}^{l} s_{13}^{l} s_{23}^{l} & c_{13}^{l} s_{23}^{l} \\
s_{12}^{l} s_{23}^{l}-c_{12}^{l} s_{13}^{l} c_{23}^{l} & -c_{12}^{l} s_{23}^{l}-c_{23}^{l} s_{13}^{l} s_{12}^{l} & c_{13}^{l} c_{23}^{l}
\end{array}\right)^{\dagger} \times\left(\begin{array}{ccc}
c_{13}^{v} c_{12}^{v} & c_{13}^{v} s_{12}^{v} & s_{13}^{v} \\
-c_{23}^{v} s_{12}^{v}-c_{12}^{v} s_{13}^{v} s_{23}^{v} & c_{12}^{v} c_{23}^{v}-s_{12}^{v} s_{13}^{v} s_{23}^{v} & c_{13}^{v} s_{23}^{v} \\
s_{12}^{v} s_{23}^{v}-c_{12}^{v} s_{13}^{v} c_{23}^{v} & -c_{12}^{v} s_{23}^{v}-c_{23}^{v} s_{13}^{v} s_{12}^{v} & c_{13}^{v} c_{23}^{v}
\end{array}\right) .
$$

This new mixing pattern for suitable value of $\lambda, a$ and $b$ is the new mixing pattern deviated from BM and HM and meet with the recent oscillation data. Values of mixing angles for the pattern along with the suitable parameters are listed in the Table 4 in case of HM, BM as well as TBM.

\section{Conclusion}

Tribimaximal mixing provides a very close description of neutrino mixing angles. However the present hint of nonzero $\theta_{13}$ coming from analysis of global neutrino oscillation data may indicate that it is broken. Here in this work, we try to use the charge lepton correction in PMNS matrix to get which can predicts recent neutrino oscillation parameters. Different neutrino mixings e.g. $\mathrm{TBM}, \mathrm{BM}, \mathrm{HM}$ as well as tetragonal mixing are well established and can explain the different pattern of neutrino in context of their mass. Analysis on these mixing are very mass important to give comments on two important aspects e.g. neutrino mass hierarchy and non-zero $\theta_{13}$. In this work we have tried to show quasi-degenerate $(\mathrm{NH}, \mathrm{IH})$ property of neutrino by parameterized standard form of neutrino mass matrix using $\Delta m_{21}^{2}, \Delta m_{31}^{2}$ and $\sum_{i} m_{i}$ considering neutrino is tribimaximaly mix. Then the deviated mixing pattern from TBM, BM and HM have been constructed using charged lepton corrections. From this analysis it can be conclude that neutrino can mix tribimaximaly, hexagonally, and bimaximaly which can predicts the neutrino oscillation parameters accurately in $3 \sigma$ level with a small correction in charged lepton part. There is a good scope for extension of this work with CP violating phases. By using $\Delta m_{21}^{2}, \Delta m_{31}^{2}$ and $\sum_{i} m_{i}$ it is also possible to get non-zero $\theta_{13}$ and solar and atmospheric mixing angle directly in the range of experimental values.

\section{Acknowledgements}

Author would like to thank Werner Rodejohann of MaxPlanck Institute, Germany for useful discussion during this work. MKD is also supported by start-up grant of Tezpur University, Tezpur.

\section{REFERENCES}

[1] K. Nakamura, et al., "Review of Particle Physics," Journal of Physics G: Nuclear and Particle Physics, Vol. 37, No. 7A, 2010, Article ID: 075021. doi:10.1088/0954-3899/37/7A/075021

[2] P. F. Harrison, D. H. Perkins and W. G. Scott, "Tri-Bimaximal Mixing and the Neutrino Oscillation Data," Physics Letters B, Vol. 530, No. 1, 2002, pp. 167-173.

[3] Z. Z. Xing, "A Full Determination of the Neutrino Mass Spectrum from Two-Zero Textures of the Neutrino Mass Matrix," Physics Letters B, Vol. 533, No. 1-2, 2002, pp. 85-90. doi:10.1016/S0370-2693(02)02062-2

[4] X. G. He and A. Zee, "Some Simple Mixing and Mass Matrices for Neutrinos," Physics Letters B, Vol. 560, No. 1-2, 2003, pp. 87-90. doi:10.1016/S0370-2693(03)00390-3

[5] G. L. Fogli, et al., "Hints of $\theta_{13}>0$ from Global Neutrino Data Analysis," Physics Letters B, Vol. 101, No. 14, 2008, Article ID: 141801 . doi:10.1103/PhysRevLett.101.141801

[6] M. C. Gonzalez-Garcia, M. Maltoni and J. Salvodo, "Updated Global Fit to Three Neutrino Mixing: Status of the Hints of $\theta_{13}>0$," Journal of High Energy Physics, No. 4, 2010, pp. 56-76.

[7] T. Schwetz, M. Tortola and J. W. F. Valle, "Where We 
Are on $\theta_{13}$ : Addendum to 'Global Neutrino Data and Recent Reactor Fluxes: Status of Three-Flavor Oscillation Parameters'," New Journal of Physics, Vol. 13, 2011, Article ID: 063004. doi:10.1088/1367-2630/13/10/109401

[8] K. Abe, et al., "Indication of Electron Neutrino Appearance from an Accelerator-Produced Off-Axis Muon Neutrino Beam," Physical Review Letters, Vol. 107, 2011, Article ID: 041801.

[9] L. Whitehead, et al., "Recent Results from MINOS, Joint Experimental-Theoretical Seminar," Joint ExperimentalTheoretical Seminar, Fermilab, 24 June 2011.

[10] C. H. Albright, A. Dueck and W. Rodejohann, "Possible Alternatives to Tri-Bimaximal Mixing," The European Physical Journal C, Vol. 70, No. 4, 2010, pp. 1099-1110. doi:10.1140/epjc/s10052-010-1492-2

[11] Z. Xing, "Tetramaximal Neutrino Mixing and Its Implications on Neutrino Oscillations and Collider Signatures," Physical Review D, Vol. 78, No. 1, 2008, Article ID: 011301. doi:10.1103/PhysRevD.78.011301

[12] W. Grimus and L. Lavoura, "A Model for Trimaximal Lepton Mixing," Journal of High Energy Physics, No. 9, 2008, p. 106. doi:10.1088/1126-6708/2008/09/106
[13] S. Goswami, S. T. Petcov, S. Ray and W. Rodejohann, "Large $\left|U_{e 3}\right|$ and Tribimaximal Mixing," Physical Review $D$, Vol. 80, No. 5, 2009, Article ID: 053013. doi:10.1103/PhysRevD.80.053013

[14] M. K. Das, M. Patgiri and N. N. Singh, "Numerical Consistency Check between Two Approaches to Radiative Corrections for Neutrino Masses and Mixings," Pramana-Journal of Physics, Vol. 65, No. 6, 2006, pp. 995-1013.

[15] N. N. Singh, M. Patgiri and M. K. Das, "Discriminating Neutrino Mass Models Using Type-II See-Saw Formula," Pramana-Journal of Physics, Vol. 66, No. 2, 2006, pp. 361-375.

[16] G. Altarelli and F. Feruglio, "Discrete Flavor Symmetries and Models of Neutrino Mixing," Reviews of Modern Physics, Vol. 82, 2010, pp. 2701-2729.

[17] S. Boudjemaa and S. F. King, "Deviations from Tribimaximal Mixing: Charged Lepton Corrections and Renormalization Group Running," Physical Review D, Vol. 79, No. 3, 2009, Article ID: 033001. doi:10.1103/PhysRevD.79.033001 